\title{
The Effect of the Solar Cycle on the Resonant Coupling of g Modes
}

\author{
C. Jordinson
}

Institute of Astronomy, University of Cambridge, UK

D. O. Gough

Institute of Astronomy and DAMTP, University of Cambridge, UK

\begin{abstract}
We consider the effect of the solar cycle on g-mode parametric resonance, and hence on the limiting amplitude of the overstable solar $g_{1}(\ell=1)$ mode. We find no change in the expected limiting amplitude from that found by Dziembowski (1983), who ignored cycle variations.
\end{abstract}

\section{Introduction}

The amplitude of the overstable $g_{1}(\ell=1)$ mode in the Sun is limited by 3 -wave resonances with higher-order modes. Varying the frequency of the overstable mode in time (due to the solar cycle) reduces the effectiveness of this resonance, and so the overstable mode might be permitted to grow to an amplitude large enough to have interesting effects in the solar core (Dilke \& Gough 1972). The limiting amplitude depends on the coupling between the modes, and is an increasing function of the daughter-modes' damping rates.

\section{Outcome}

Taking account of the frequency changes due to the solar cycle, the limiting amplitude of the parent (overstable) mode due to a given pair of daughter modes is increased by a factor of order the inverse of the fractional time spent at resonance (i.e. the fraction of the time when the frequencies of the modes obey $\left|\omega_{1}-\omega_{2}-\omega_{3}\right|<\gamma$, where $\gamma$ is the decay rate of the daughter modes). However, the vacillating frequency of the parent mode increases the probability of there being a resonance with daughter modes in a less dense part of the spectrum, in which the damping rates are smaller, hence giving a lower limiting amplitude. The two effects cancel, leading to no change in the expected limiting amplitude from that found by Dziembowski (1983).

\section{References}

Dilke, F. W. W. \& Gough, D. O. 1972, Nature, 240, 262

Dziembowski, W. 1983, Solar Phys., 82, 259 